\title{
Brorfelde Schmidt CCD Catalog (BSCC)
}

\author{
N. Zacharias ${ }^{1}$, O. H. Einicke², K. Augustesen ${ }^{2}$, J. V. Clausen², C. Finch ${ }^{1}$, E. Høgㄹ, G. L. \\ $\mathrm{Wycoff}^{1}$ \\ nz@usno.navy.mil \\ ${ }^{1}$ U.S. Naval Observatory, 3450 Mass.Ave. NW, Washington DC 20392; \\ ${ }^{2}$ Niels Bohr Institute, Cøpenhagen University, Juliane Maries Vej 30, DK-2100 Cøpenhagen Oe, Denmark
}

\begin{abstract}
The Brorfelde Schmidt CCD Catalog (BSCC) contains about 13.7 million stars, north of $+49^{\circ}$ Declination with precise positions and V, R photometry. The catalog has been constructed from the reductions of 18,667 CCD frames observed with the Brorfelde Schmidt Telescope between 2000 and 2007. The Tycho-2 catalog was used for astrometric and photometric reference stars. Errors of individual positions are about 20 to 200 mas for stars in the $\mathrm{R}=10$ to 18 mag range. External comparisons with 2MASS and SDSS reveal possible small systematic errors in the BSCC of up to about 30 mas. The catalog is supplemented with $\mathrm{J}, \mathrm{H}$, and $\mathrm{K}_{s}$ magnitudes from the 2MASS catalog.

Subject headings: astrometry — catalogs — methods: data analysis
\end{abstract}

\section{INTRODUCTION} ibration frames were sent to the U.S. Naval was used with a $2 \mathrm{k}$ by $2 \mathrm{k}$ charge-coupled device (CCD) camera (Florentin-Nielsen \& Jørgensen 1995) to observe the sky from $+49.6^{\circ}$ to $+90^{\circ}$ declination, with additional observations taken in selected fields north of $-10^{\circ}$. The main idea of the project was to supplement the Carlsberg Meridian Circle Catalog (CMC14) (1), which covers the $-30^{\circ}$ to $+50^{\circ}$ declination area. However, the resulting Brorfelde Schmidt CCD Catalog (BSCC) does not have a complete sky coverage north of $+49.6^{\circ}$, with about $30 \%$ of that area not observed. The sky coverage of the BSCC is shown in Figure 1. This catalog will be very valuable for determination of highly accurate proper motions of faint stars when combining with future data, as for example the upcoming USNO Robotic Telescope (URAT) survey (Zacharias 2008) and for assisting in systematic error analysis of existing catalogs like the UCAC3 (Zacharias et al. 2010) and PPMXL (Roeser et al. 2010).

The CCD data were obtained during the period of 2000 to 2007 and all applicable 18,667 object CCD frames together with additional cal1964tervatory (USNO) in 2009 for processing. The USNO CCD Astrograph Catalog (UCAC) project (Zacharias et al. 2010) reduction pipeline (Zacharias 2010) was modified and used for this project. A catalog of 13.7 million stars for the magnitude range $\mathrm{R}=10$ to 18 has been constructed with precise positions on the International Celestial Reference System (ICRS) by use of the Tycho-2 (Høg et al. 2000) reference star catalog.

Photometry in $\mathrm{V}$ and $\mathrm{R}$ has been obtained for most of these stars from the CCD data, with Twomicron All Sky Survey (2MASS) near-infrared photometry added to the catalog (2). The filters used at the Brorfelde Schmidt for this project are approximating the standard Johnson $\mathrm{V}$ and Cousin R filters.

Details about the data acquisition and astrometric and photometric reductions are presented here, followed by a description of the resulting catalog. External comparisons were performed with the Sloan Digital Sky Survey (SDSS) release 7 data (www.sdss.org/DR7/) and the 2MASS point 
source catalog (www.ipac.caltech.edu/2mass). The BSCC is available from the Strasbourg Data Center (CDS).

\section{OBSERVATIONS}

The observations were performed during 328 nights between 2000 February 13 and 2007 September 6 at the (now closed) 50/77 cm Brorfelde Schmidt Telescope belonging to Copenhagen University. It is located at $55^{\circ} 37^{\prime} 31^{\prime \prime} \mathrm{N}, 11^{\circ} 39^{\prime}$ 59 " E. Its focal length, $150 \mathrm{~cm}$, and thereby its focal plane scale of $137 \mathrm{arcsec} / \mathrm{mm}$ was chosen to match the typical atmospheric seeing in Brorfelde of 2 arcsec and match the resolution of available photographic emulsions.

Since 1999, the telescope has been equipped with a 2048x2048 thick, front side illuminated Kodak KAF-4201 CCD, which is cooled by a threestage Peltier element, which again is water cooled (Florentin-Nielsen \& Jørgensen 1995). The pixel size of 9 microns yields a scale of $1.24 \mathrm{arcsec} / \mathrm{pixel}$ and a field size of $42 \times 42$ arcmin.

For the present project, exposures were obtained through B, V, R, and I filters, respectively, with transmission curves shown in Figure 2. Exposure times were 60 to $600 \mathrm{sec}(\mathrm{B}), 10$ to $600 \mathrm{sec}$ $(\mathrm{V}, \mathrm{R})$, and 90 to $600 \mathrm{sec}(\mathrm{I})$, with the majority of data being $90 \mathrm{sec} \mathrm{V}$ and $\mathrm{R}$ images. All exposures were taken close to the meridian with maximum deviations of $-12^{\circ}$ and $+10^{\circ}$, respectively. Bias and dark exposures were taken each night, and dome flat exposures were secured at regular intervals during daytime.

\section{DATA REDUCTION}

All data reduction was performed in the Cataloging Division of the U.S. Naval Observatory, using DS9 and IRAF for manual investigation of FITS CCD frames and custom Fortran software for all pipeline processing, based on the code used for the UCAC project.

\subsection{Pixel Data Processing}

Without assuming metadata to be correct, all available CCD data FITS files were read with custom utility code to derive statistics about pixel value histograms and to retrieve header information. Visual inspection of the resulting output ta- bles showed that in most cases the first bias frame taken at the beginning of each night displays a large number of bright pixels. All first images of any given night were thus excluded from the following processing. Depending on the statistical properties of the CCD frames they were identified and grouped into bias, darks, flat, and science object files. A total of 25,190 CCD frames were provided by Copenhagen University, of which 2,884 were identified as bias, 2,245 as dark, 271 as flat, and 18,926 as science frames. A total of 60 frames failed the automated classification and manual inspection revealed overexposed flats, which were disregarded. The 804 binned data frames were also not processed.

Dark frames were utilized to identify "bad" pixels which were flagged and excluded from further processing. For each standard exposure time combined darks were derived. For exposure times which were not used often a single master dark was calculated from all available dark frames of that exposure time. For the 20, 25, and 60 second exposures, master darks by observing year were generated, while for the most often used 90 second exposures a combined dark per month of observing was established. A single bias-offset value per individual dark frame (added to all pixels of a frame; a different value for different frames) was applied to bring all frames to the same bias level value before combining. However, individual frames were not corrected for bias frame subtraction because the bias level structure was found to be well corrected with applying combined darks.

A combined, master flat file was created for each filter and each year of observing from the available dome flat data, excluding overexposed frames. The appropriate master dark frames were subtracted from the flats before combining. Individual flat frames were normalized, low/high pixel values excluded, and the mean taken over the remaining values for each pixel to arrive at a combined flat.

All combined flats were analyzed to identify outlier pixel values and largely variable pixels. Those were added to the "bad pixel" map and not used in the astrometric and photometric reductions of stellar images. 


\subsection{Astrometric Reductions}

Science frames were grouped by filter and reduced separately. Tycho-2 reference stars were identified in the $x, y$ data and weighted, leastsquares, conventional "plate" adjustments (CPA) run on all applicable frames. A total of 127 I, 9350 $\mathrm{R}, 9115 \mathrm{~V}$, and $75 \mathrm{~B}$ filter frames provided successful solutions (18,667 CCD frames all together). Of these, a total of 1,888 CCD frames are outside the $+49.6^{\circ}$ to $+90^{\circ}$ area which constitutes the published catalog data, thus 16,779 CCD frames were used for the BSCC. The number of reference stars used per CCD frame is shown in the histogram of Fig. 3. There are 427 frames with 9 or less reference stars in the area north of $+49.5^{\circ}$, while the typical number of Tycho-2 reference stars used per frame is about 12 to 35 , with a few frames up to about 100 .

A linear plate model (6 parameters) was adopted without correcting for differential refraction or aberration. Also no third order geometric distortion was pre-applied, which is inherent in Schmidt telescope imaging (curved focal plane and tangential projected $\xi, \eta$ ). These procedures are adequate due to the small field of view and the presence of other distortions for example from the filters. However, the combined effect of all geometric distortions is determined empirically as follows, and has been corrected.

A total of 312,522 and 292,957 residuals (with 2 coordinates each) were available from the astrometric reductions of all $\mathrm{R}$ and $\mathrm{V}$ filter observations, respectively. The residuals were stacked up in bins as function of focal plane $x, y$ coordinates and slightly smoothed by weighted average with neighboring bins. The resulting field distortion patterns (FDP) are shown in Fig. 4. Linear interpolation between the bins were performed to arrive at the FDP correction values which were applied to the $x, y$ data prior to the following iteration of the CPA reduction.

Residuals of the final CPA reductions were plotted as a function of $x, y$ coordinate, radial distance from the frame center, magnitude, color, and coma term (product of magnitude and coordinate). The largest systematic errors, up to about 20 mas, were found as a function of magnitude (Figs. 5, 6), with root-mean-square (RMS) scatter shown in Fig. 7. For most of these $90 \mathrm{sec}$ exposures in the survey saturation is around $\mathrm{R}=10$ preventing from reaching even smaller positional errors at the bright end. Errors for stars fainter than 10th magnitude are dominated by the reference star contribution. A complex, non-linear dependence for the $y$ coordinate (declination) as a function of coma-x and coma-y is found (not shown) with amplitudes up to 20 mas. These systematic errors are at the limit of the reference star catalog at the epoch of the CCD observations. No further investigation was performed and no attempt to correct the derived star positions for any such magnitude dependent systematic errors was made.

The final catalog positions are obtained from a weighted mean of all individual observations for each star. Objects matching within 2.0 arcsec are assumed to be the same star. Thus the positions provided in this observational catalog are based on the mean of the $\mathrm{V}$ and $\mathrm{R}$ band observations, given at mean epoch of observation, on the ICRS, as represented by the Tycho- 2 catalog.

\subsection{Photometric Reductions}

Differential photometric reductions were performed with respect to Tycho-2 standards on individual CCD frames. Instrumental magnitudes were derived from the integral (volume) of the fitted stellar image profiles above the local background using the modified UCAC reduction pipeline while performing the astrometric pixel reduction step. In the following, only Tycho-2 stars with "good" photometry flag, brighter than $\mathrm{V}=12.5$, and not saturated on CCD frames were used. Furthermore, stars with $(\mathrm{B}-\mathrm{V}) \leq-0.2$ and $(\mathrm{B}-\mathrm{V}) \geq 1.5$ were excluded and stars in the $(\mathrm{B}-\mathrm{V})$ range of 1.1 to 1.5 were used with reduced weight.

A magnitude zero-point $\left(V_{0}\right)$ per V-band CCD frame was fitted to transform the instrumental magnitudes $\left(V_{i}\right)$ into standard $\mathrm{V}$ magnitudes $\left(V_{s}\right)$, using Tycho-2 references for $V_{s}$ in a weighted leastsquares adjustment according to

$$
V_{0}=V_{s}-V_{i}
$$

Similarly,

$$
R_{0}=R_{s}-R_{i}
$$


was determined using $\mathrm{B}$ and $\mathrm{V}$ magnitudes from Tycho-2 as standards with the approximation

$$
R_{s} \approx V_{s}-s(B-V)
$$

The color slope term, $s=0.45$, was derived from linear fits to sample CCD data with many reference stars and solving for both the slope and constant term. The average resulting slope term was adopted and used to solve only for the photometric zero-point constant in all R-band CCD frames.

Fig. 8 shows the distribution of the standard error of this photometric constant. For most frames this error is 20 to 40 millimag. The absolute photometric error of stars in the BSCC has to be larger than this photometric constant error, while the internal, photometric precision is better for well exposed stars.

\section{RESULTS AND EXTERNAL COM- PARISONS}

\subsection{The Catalog}

The BSCC contains 13,771,775 stars north of $+49.6^{\circ}$ declination and is sorted by declination. Of these, 13,157,292 do have a match with a 2MASS star within 2 arcsec, and $\mathrm{J}, \mathrm{H}, \mathrm{K}_{s}$ photometry with errors were copied from the 2MASS into BSCC. Stars based on a single CCD observation and not matched with 2MASS did not enter the released catalog. Fig. 9 shows the distribution of stars in the BSCC by R and V magnitude. Completeness is expected up to about $\mathrm{R}=17.5$ with a limiting magnitude of about $\mathrm{R}=19$. There are 583,043 stars in the catalog without $\mathrm{R}$ magnitude, and 4,311,081 do not have a $\mathrm{V}$ magnitude.

The BSCC data file is $1.7 \mathrm{~GB}$, formatted, ASCII. Some sample lines are listed in Table 1 , with the data format explained in Table 2 . This is an observational catalog of mean positions at a mean epoch, which is slightly different for each star. The positions are on the International Celestial Reference System (ICRS) by means of the Tycho- 2 reference star catalog. There are no proper motions provided. Stars are identified by the IAU registered acronym "BSCC", followed by a fixed-length, 8-digit running record number without a space.
Fig. 10 shows the mean astrometric errors (per coordinate) of the BSCC as a function of R magnitude. The filled circles show the errors as derived from the model (including $x, y$ fit precision and expected contribution from the "plate" adjustment solution), while the open squares show the error from the observed scatter of individual positions. These are small number statistics for individual stars (with typically 2 to 4 images) but become meaningful when averaged over many stars (for the $\mathrm{R} \geq 9$ range). Many stars in the 10 to $15 \mathrm{mag}$ range have internal errors of about 20 mas in Dec and about 25 mas in RA. A near saturation effect seems to be present for stars brighter than $\mathrm{R}=11$ mag showing a slightly larger, observed, scatter error than at $\mathrm{R}=11 \mathrm{mag}$.

Fig. 11 shows the formal, photometric errors in the BSCC R and V magnitudes derived from the scatter of individual magnitudes. Data shown for stars brighter than about 9th mag are affected by small number statistics. Systematic errors are not included here. The precision of the photometry is on the 3 to $5 \%$ level for the 10 to 15 mag range, then increasing according to the lower signal-tonoise ratio to about 0.15 mag at $\mathrm{R}=18$.

\subsection{Astrometric Comparisons}

Unweighted position differences of BSCC minus Tycho-2, averaged over 100 stars per dot are shown in Fig. 12 as a function of magnitude. Tycho-2 proper motions have been applied to bring the positions to the epoch of individual BSCC stars. The small overall offset in declination is caused by the weighted adjustment in the CCD reductions of individual frames combined with the magnitude dependent pattern shown in Fig. 6. The differences in RA average to about zero, as expected.

Position differences of BSCC with respect to 2MASS are shown in Fig. 13. No proper motions have been applied because neither the 2MASS nor the BSCC do have proper motions. The epoch difference between individual BSCC and 2MASS observations of a star is within \pm 3 years. Over 11 million stars were matched. There is a small systematic difference along RA increasing toward faint stars reaching about 30 mas at $R=18$, while for Dec there is a remarkable consistency between the BSCC and 2MASS data with only about 10 mas differences at the very faint end at $R=18$ and 
systematic differences less than about 5 mas for the entire magnitude range of $\mathrm{R}=10$ to 17 .

Fig. 14 shows the position differences BSCC-2MASS as a function of declination. CCD frame size patterns are clearly seen with systematic position differences of up to about 50 mas. Some of this is caused by not having proper motions applied. The break at $\delta=60^{\circ}$ is explained by the mean epoch of BSCC observations (Fig. 15). Data for $\delta<60^{\circ}$ were observed, on average, at a much later epoch than the other data, resulting in a larger epoch difference to 2MASS data. This in turn gives larger position differences because no proper motions are applied. The data for $\delta \geq 78^{\circ}$ were observed at a mid-range epoch, leading also to an increased scatter in the position differences as compared to data between $60^{\circ}$ and $78^{\circ}$. Fig. 16 shows the BSCC-2MASS position differences as a function of right ascension. The average offset for the RA component is a result from the magnitude equation seen earlier (Fig. 13). In addition, a sine wave pattern of about 20 mas amplitude is present in either coordinate. This could be caused by galactic dynamics due to the lack of proper motions in this comparison.

A representative subset of the Sloan Digital Sky Survey (SDSS) release 7 data (Abazajian et al. 2009), (Munn et al. 2004) was selected $\left(200^{\circ} \leq \alpha \leq\right.$ $\left.270^{\circ},+58^{\circ} \leq \delta \leq+90^{\circ}\right)$. A match with BSCC resulted in 49,692 common stars and SDSS proper motions were applied. The position differences BSCC-SDSS are shown in Figs. 17 and 18 as functions of magnitude and color, respectively. The RA coordinate shows a small magnitude equation, about $5 \mathrm{mas} / \mathrm{mag}$, similar to the BSCC-2MASS differences (Fig. 13), for the 14 to 18 mag range. The Dec coordinate displays a constant offset of about 10 mas over that magnitude range. There is no dependence of the BSCC-SDSS position differences as a function of color, except for the average offset mentioned before.

Fig. 19 shows the position differences BSCC-CMC14 as a function of $\mathrm{R}$ magnitude. No proper motions were applied and the epoch difference is only a few years. Note that the overlap between these 2 catalogs is very small, involving only $194 \mathrm{CCD}$ frames taken at $+50^{\circ}$ declination in the $\mathrm{RA}=8$ to 16 hour range with 20,596 stars in common. Fig. 20 shows the BSCC-2MASS position differences for the same area in the sky as used for the CMC14 comparison, which is significantly different than the average BSCC-2MASS differences of Fig. 12. Stars with position differences over 300 mas in either coordinate were excluded for this comparison. For the RA component, CMC14 agrees with 2MASS but both are offset with respect to BSCC by about -30 mas. For the Dec component, BSCC agrees with 2MASS but CMC14 is offset by about +30 mas with respect to the other 2 catalogs.

\subsection{Photometric Comparisons}

Fig. 21 shows the difference in BSCC R minus CMC14 r magnitude as a function of CMC14 $\mathrm{r}$ magnitude. A narrow distribution is seen with systematic differences in the range of about \pm 0.1 mag. Fig. 22 shows the color-color diagram between the BSCC V, R and SDSS $\mathrm{g}$ and $\mathrm{r}$ bandpasses. The BSCC R band magnitudes match the SDSS $r$ magnitudes very well for $(g-r)$ colors between 0.2 and 1.0, with significant systematic differences for redder stars. The BSCC V and SDSS g magnitudes are significantly different (up to 0.4 mag) for all colors, as expected.

\section{DISCUSSION}

Considering the low resolution (1.24 arcsec/pixel) of the Brorfelde Schmidt survey data the catalog is amazingly accurate, with repeatability of observations (from overlapping fields) of about 20 mas per coordinate for well exposed stars. Systematic errors in observed star positions are also small, on the 30 mas level or below, with particularly well controlled systematic errors as a function of magnitude (see for example the comparison with 2MASS data).

However, these survey data could benefit from a denser reference star catalog than the Tycho2. Most of the highly accurate Tycho stars are just overexposed in the Schmidt CCD data, while the accessible Tycho stars around magnitude 11 to 12 are affected by relatively large errors. Typical CPA solutions have a standard error of 60 to 80 mas (combined error of the $x, y$ data and the reference star positional errors, per star and coordinate). Thus the "plate" parameters are not very well defined, which leads to significant scatter in samples of a few CCD frames, as seen for example in the comparison of BSCC data with the CMC14 catalog which barely overlap at declination $+50^{\circ}$. 
A block-adjustment type solution for overlapping frames is difficult with the BSCC data due to the large gaps in the survey area coverage and not enough overlap between adjacent CCD images (no 2 or 4 -fold overlap pattern).

Similarly the photometric results suffer from a weak determination of the zero-point per CCD frame due to the small number of available, highly accurate standard stars. Once a denser photometric catalog than the Tycho-2 becomes available, the BSCC data would benefit from a re-reduction.

The most important aspect of the BSCC astrometric data is the high signal-to-noise data for stars at the faint UCAC end, i.e. stars around R $=15$ to 16 . The precision of the BSCC positions is about 40 mas per coordinate, while the UCAC data are on the 70 mas level at those magnitudes. However, only a small area of the sky could be covered with this 7-year effort. Full-sky coverage with high accuracy down to about $\mathrm{R}=17.5$ will hopefully be achieved soon with the URAT project (Zacharias 2008). Combining URAT data with the BSCC will provide excellent proper motions for those faint stars on the 3 mas/yr level, completely based on CCD observations for early and current epoch data.

We wish to thank the former director of the Copenhagen University Observatory, Henning Jørgensen, who played a key role in the definition and initialization of the project.

This publication makes use of data products from the Two Micron All Sky Survey, which is a joint project of the University of Massachusetts and the Infrared Processing and Analysis Center/California Institute of Technology, funded by the National Aeronautics and Space Administration and the National Science Foundation.

Funding for the SDSS and SDSS-II has been provided by the Alfred P. Sloan Foundation, the Participating Institutions, the National Science Foundation, the U.S. Department of Energy, the National Aeronautics and Space Administration, the Japanese Monbukagakusho, the Max Planck Society, and the Higher Education Funding Council for England. The SDSS Web Site is http://www.sdss.org/.

National Optical Astronomy Observatories (NOAO) is acknowledged for IRAF, Smithonian
Astrophysical Observatory for DS9 image display software, and the California Institute of Technology for the pgplot software.

\section{REFERENCES}

Abazajian, K. N. et al. 2009, ApJS 182, 543

Andersen, J. Clausen, J. V. 1974, A\&A, 34, 423

Carlsberg Meridian Catalogue 14 (CMC14), Copenhagen University, Institute of Astronomy Cambridge, \& Real Instituto y Observatorio de la Armada San Fernando, 2006, www.ast.cam.ac.uk/ dwe/SRF/cmc14.html, VizieR on-line Catalog I/304

Florentin-Nielsen, R. \& Jørgensen, H. E. 1995, ASP Conf. Ser. 84, 450

Høg, E. Fabricius, C., Makarov, V., Urban, S., Corbin, T., Wycoff, G., Bastian, U., Schwedendiek, P., Wicenec, A. 2000, A\&A, 355, L27

Munn, J. A., Monet, D. G., Levine, S. E. et al. 2004, AJ 127, 3034

Roeser, S. et al. 2010, in press with AJ

Skrutskie, M. F, Cutri, R. M., Stiening, R. et al. 2006, AJ, 131, 1163

Zacharias, N. 2008, in proceed. IAU Symp. 248, Eds. W. Jin, I. Platais, \&M. Perryman, p. 310, Cambridge Univ.Press

Zacharias, N. et al. 2010, AJ 139, 2184

Zacharias, N. 2010, AJ 139, 2208

This 2-column preprint was prepared with the AAS LATEX macros v5.2. 

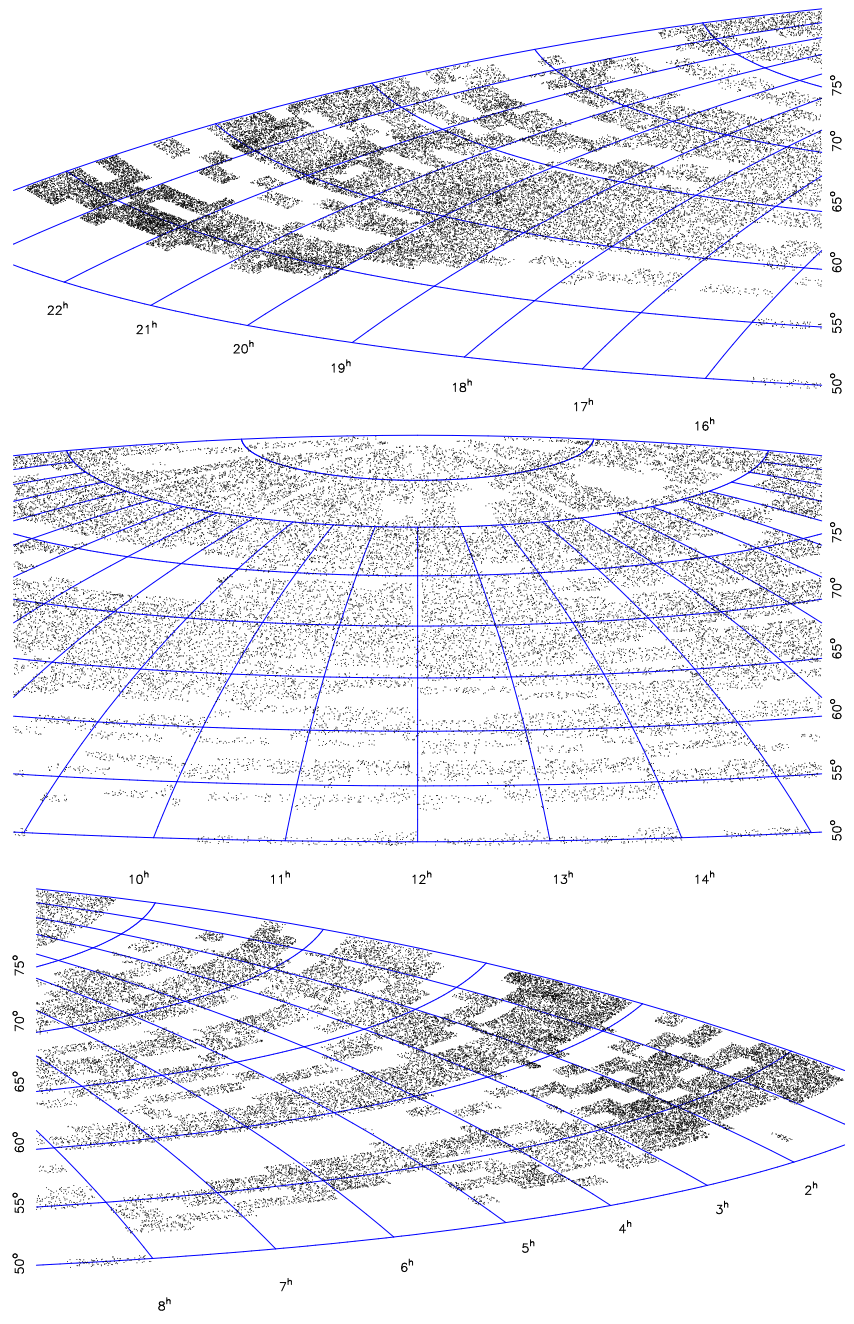

Fig. 1.- Distribution of BSCC stars on the sky. Shown are stars in the R-mag range of 10 to 12 between +49 and +90 declination in 3 sections split by RA on equal-area projections.

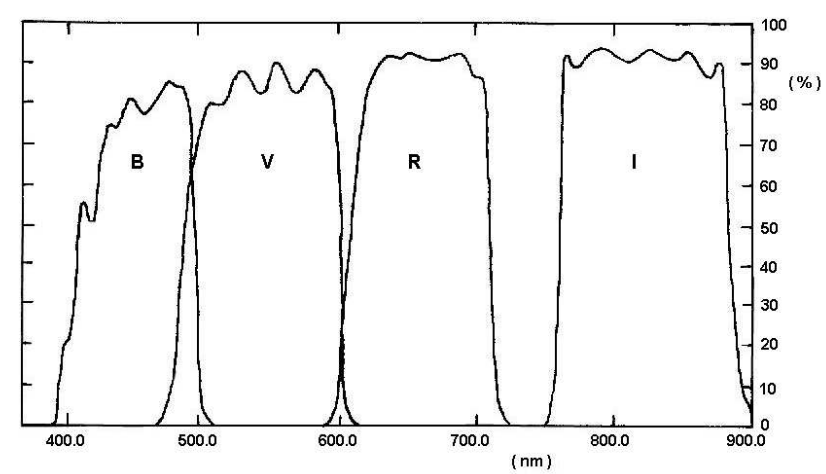

Fig. 2.- Bandpass data for the B, V, R, and I filters used at the Brorfelde Schmidt telescope for this project.

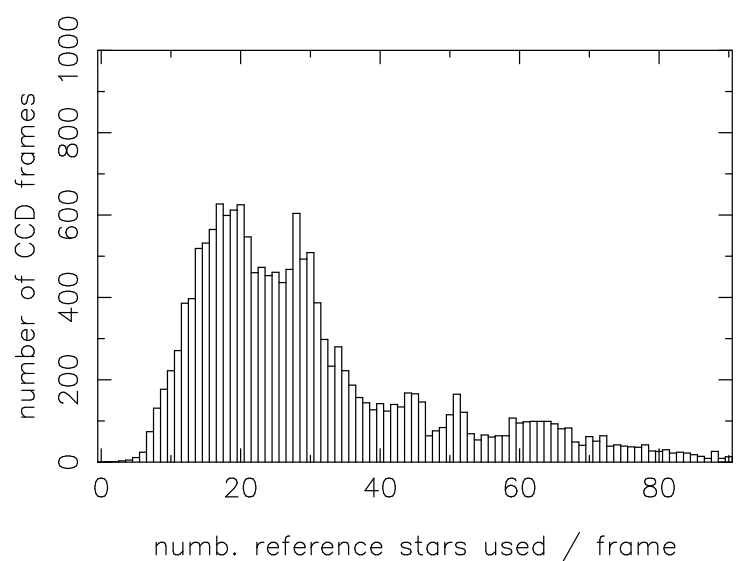

Fig. 3.- Histogram of the number of reference stars (from Tycho-2) actually used per CCD frame in the astrometric reductions of the BSCC. 
091210 BS mod=3, run, fil=023 no FDP scale $=2000$ smooth

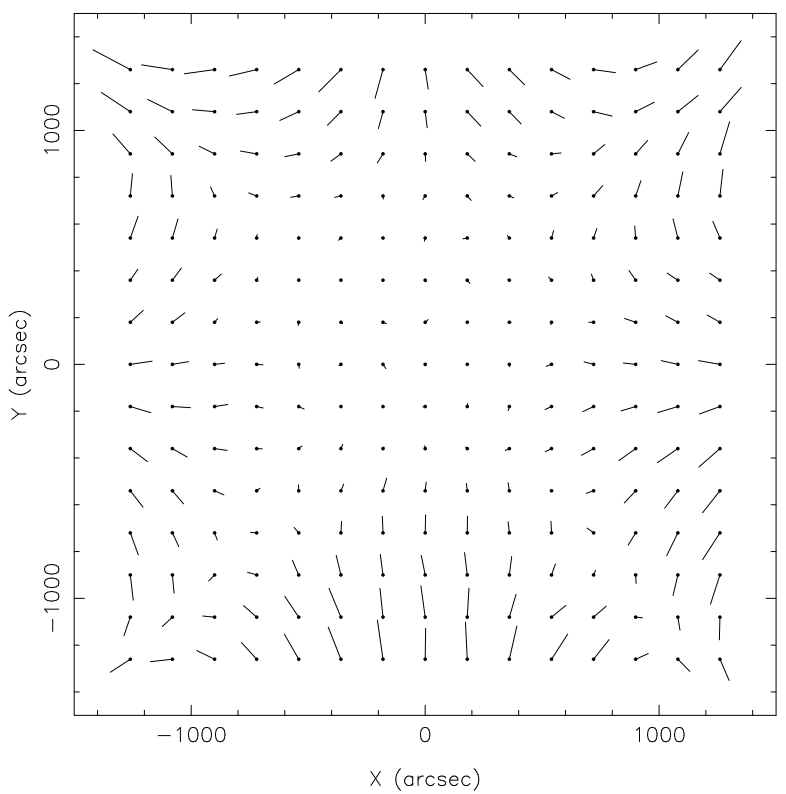

091210 BS mod=3, run, fil $=024$ no FDP scale $=2000$ smooth

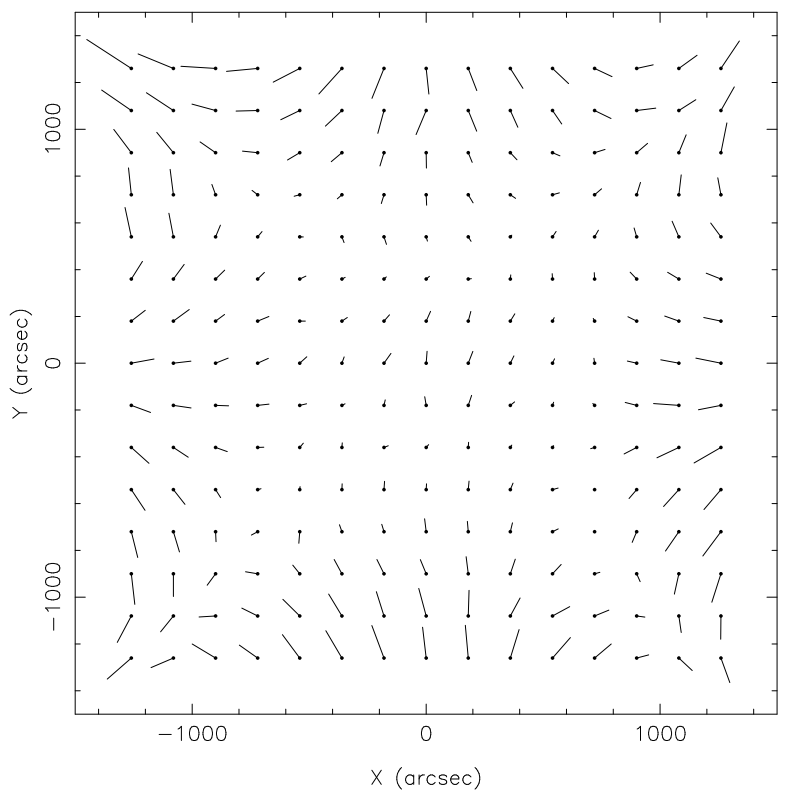

Fig. 4.- Field distortion pattern derived from the residuals of Tycho-2 reference stars for all applicable R-band (top) and V-band (bottom) Brorfelde Schmidt CCD frame observations. The scale of the residual vectors is 2000 , thus the largest vectors are about 100 mas.
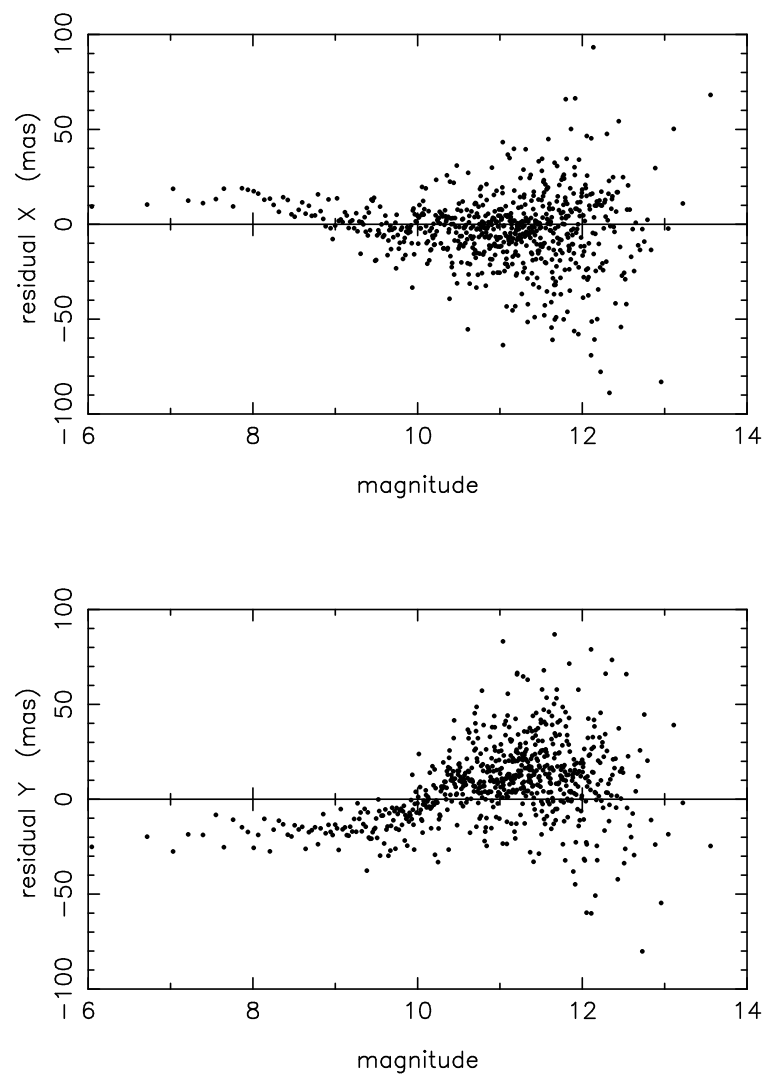

Fig. 5.- Residuals from final reductions of Rband observations with respect to Tycho- 2 reference stars as a function of magnitude. One dot represents the mean over 400 stars. 

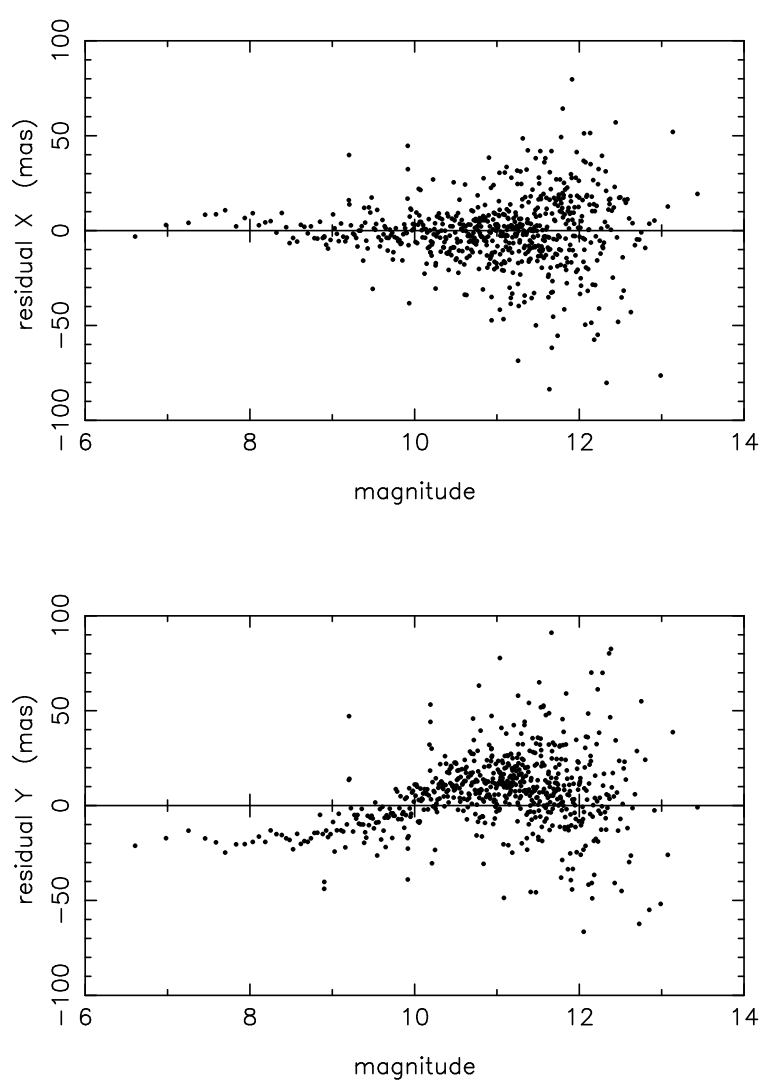

Fig. 6. - Same as the previous figure but for the V-band observations.
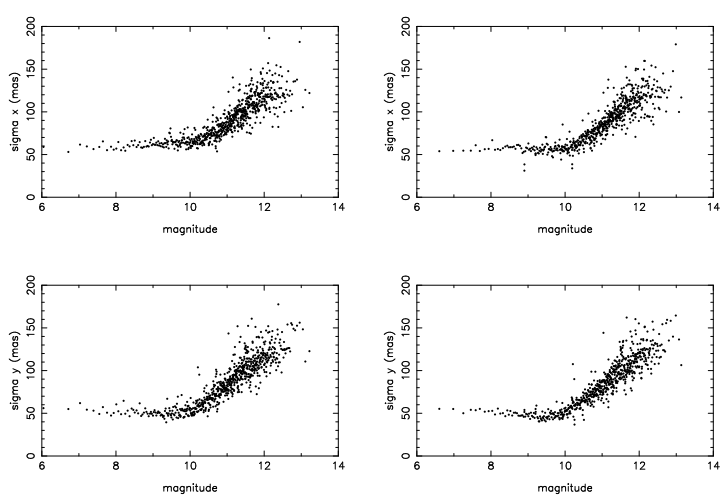

Fig. 7.- Scatter (standard deviation) of the residuals for R-band (left) and V-band (right) observations as a function of magnitude. One dot represents the mean over 400 stars.

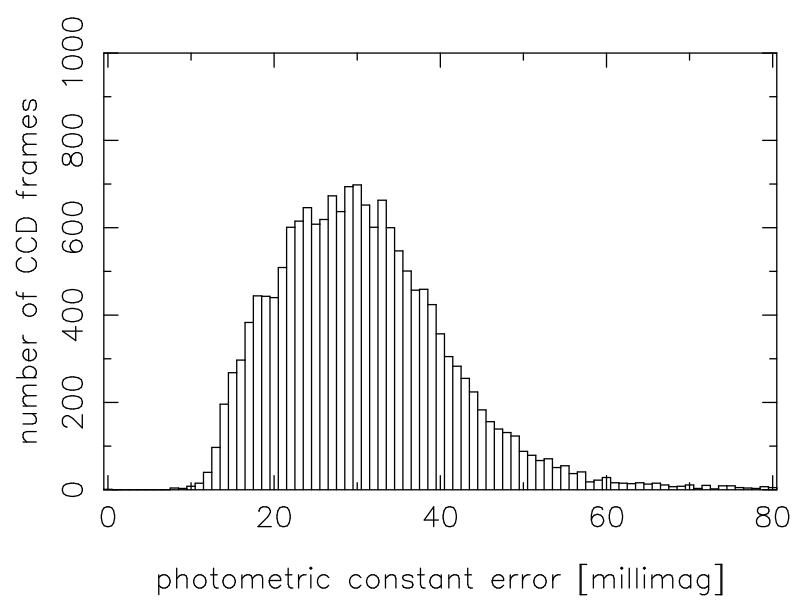

Fig. 8.- Distribution of the standard error in determining the photometric constant between instrumental and absolute magnitudes. This error varies significantly depending on the number and quality of Tycho-2 photometric standard stars available in any given CCD frame.
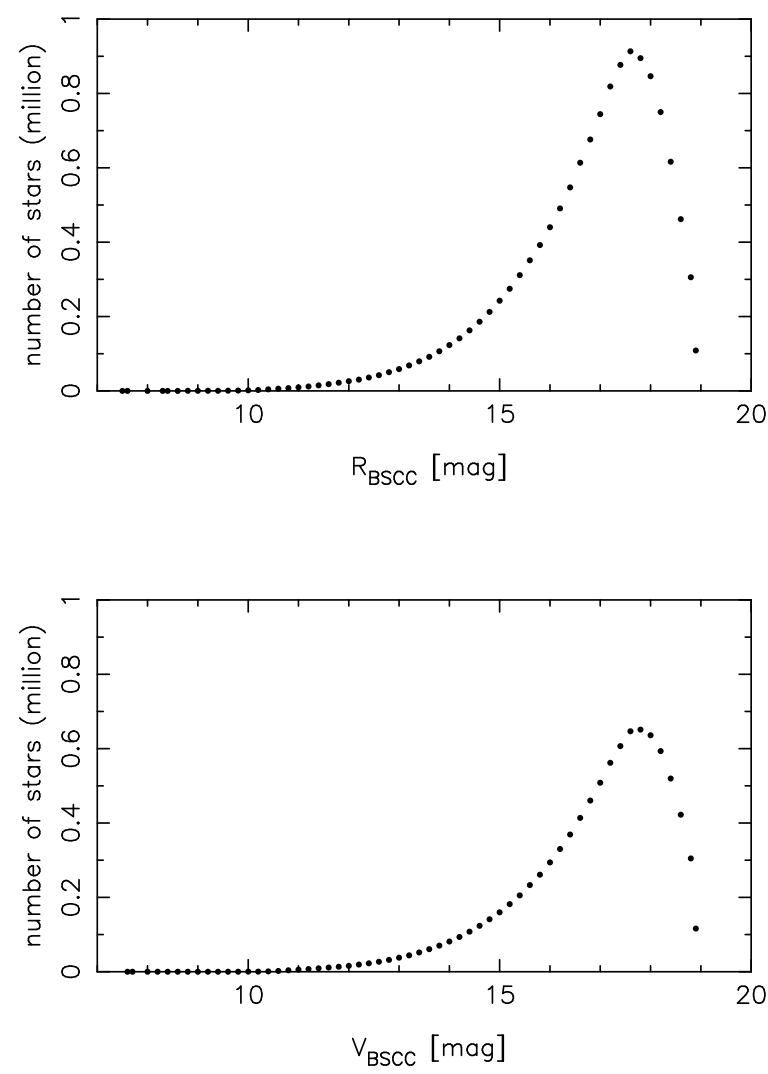

Fig. 9.- Distribution of BSCC stars by $\mathrm{R}$ and V magnitude. 

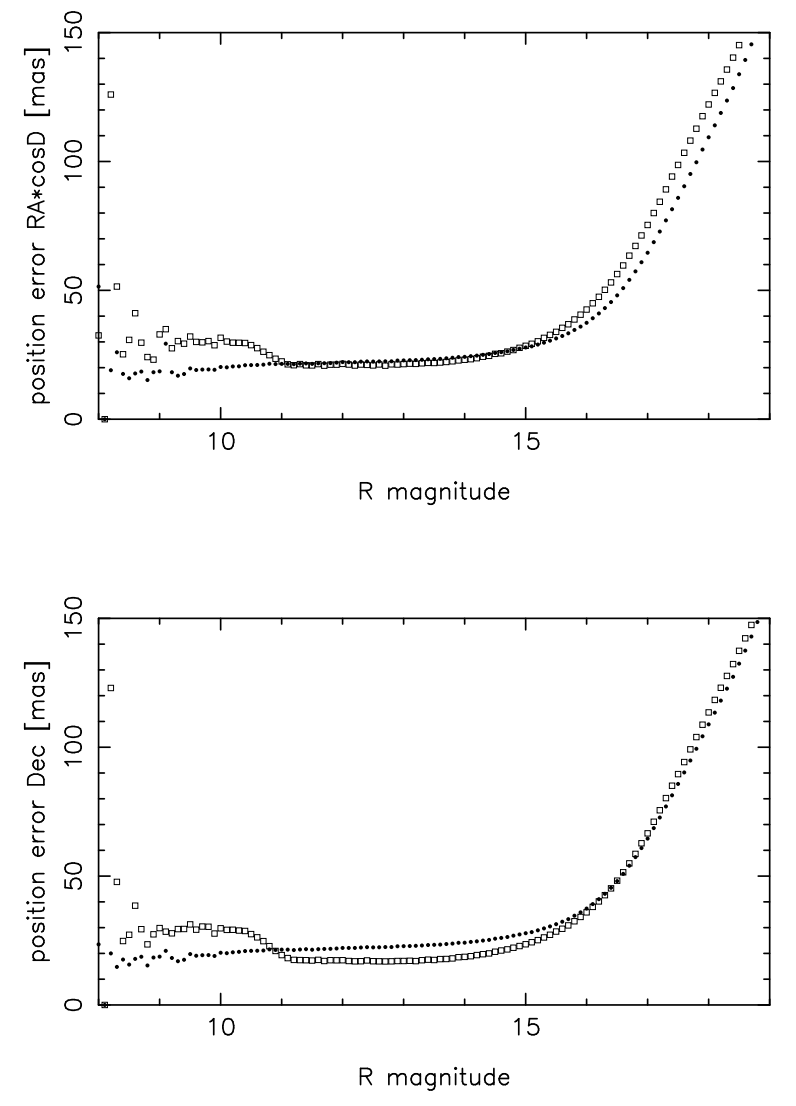

Fig. 10.- Mean positional errors as a function of $\mathrm{R}$ magnitude for the RA (top) and Dec (bottom) components. The filled circles show the model standard error, while the open squares show the mean standard error from the scatter of individual positions which contribute to a mean catalog position.

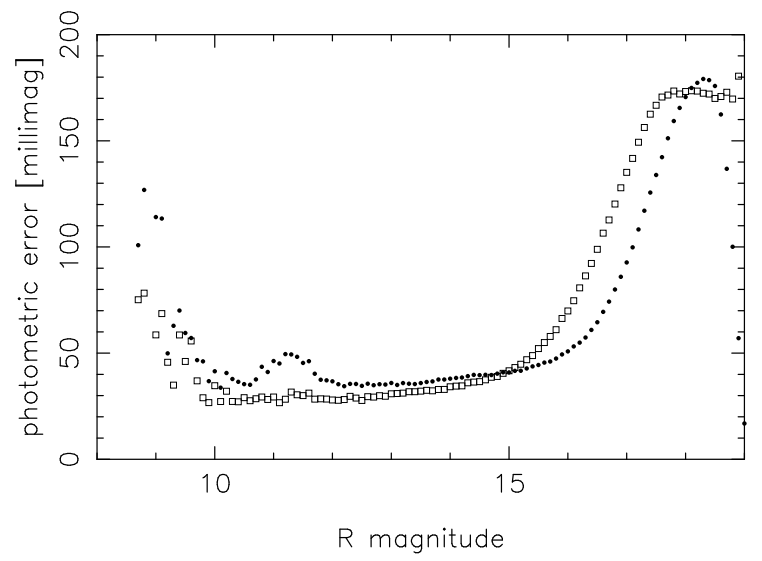

Fig. 11.- Formal error on photometry of the BSCC as a function of $\mathrm{R}$ magnitude. The filled circles are for the $\mathrm{R}$ mag error, while the open squares are for the $\mathrm{V}$ mag error.
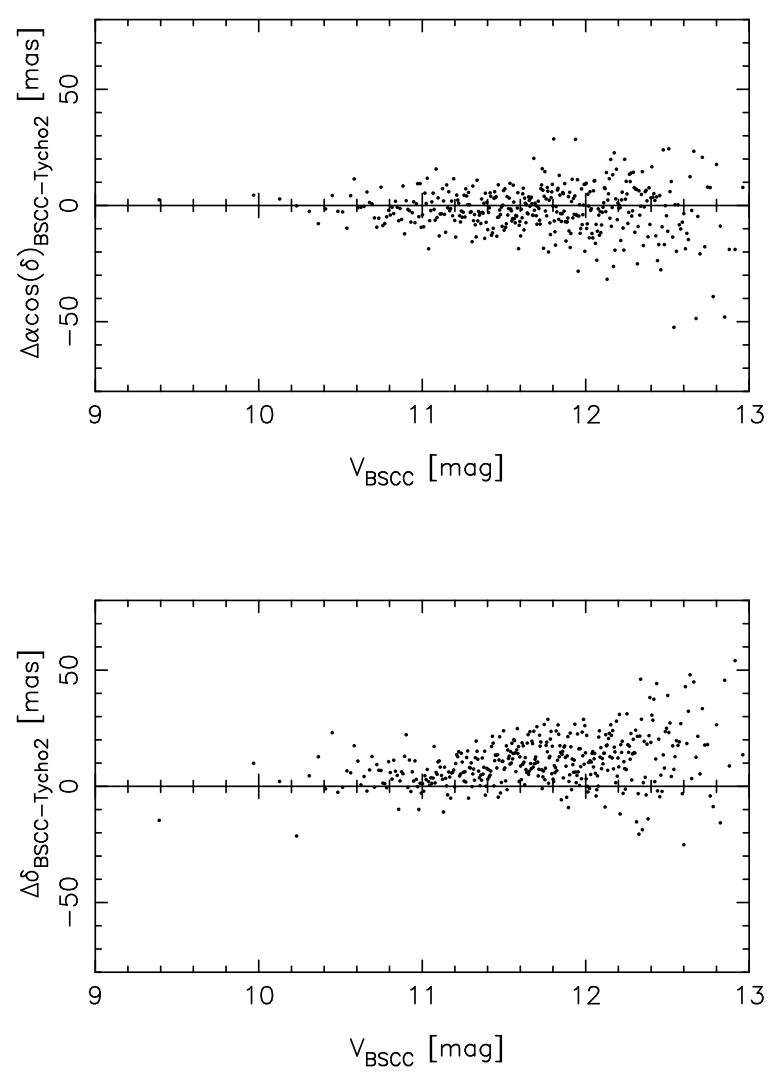

Fig. 12.- Unweighted position differences BSCC-Tycho2 in right ascension (top) and declination (bottom) as a function of $\mathrm{V}$ magnitude. One dot represents the mean over 200 stars. 

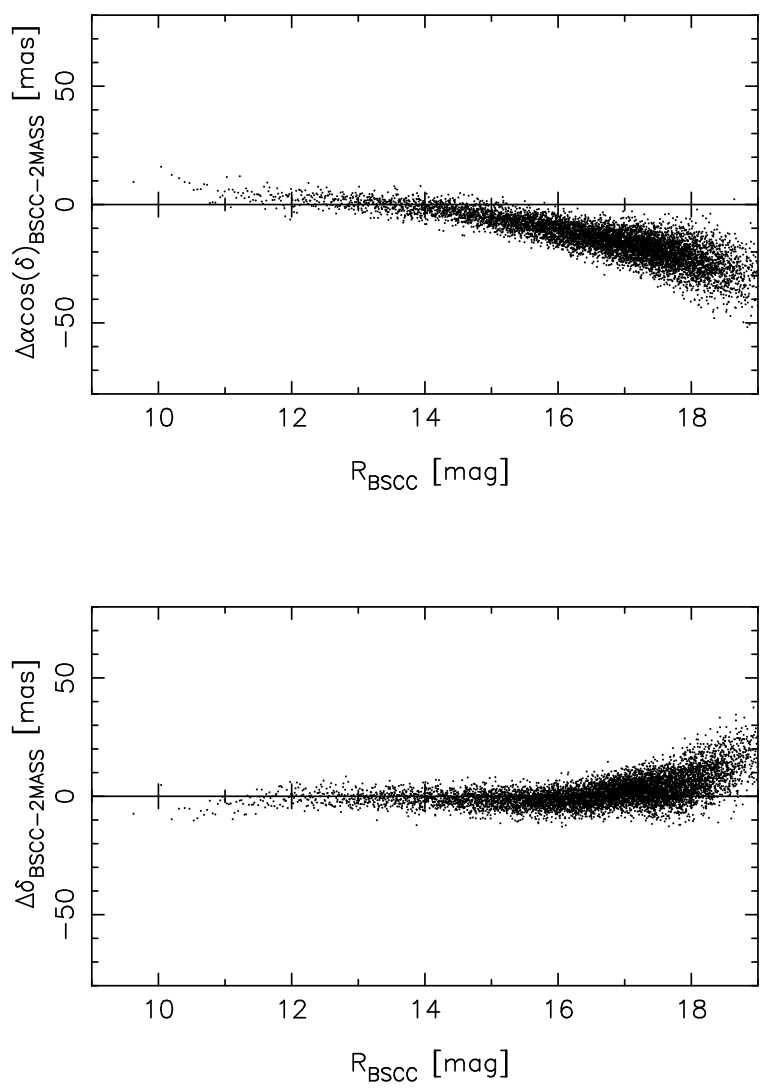

Fig. 13.- Position differences BSCC-2MASS in right ascension (top) and declination (bottom) as a function of $\mathrm{R}$ magnitude. One dot represents the mean over 1500 stars.
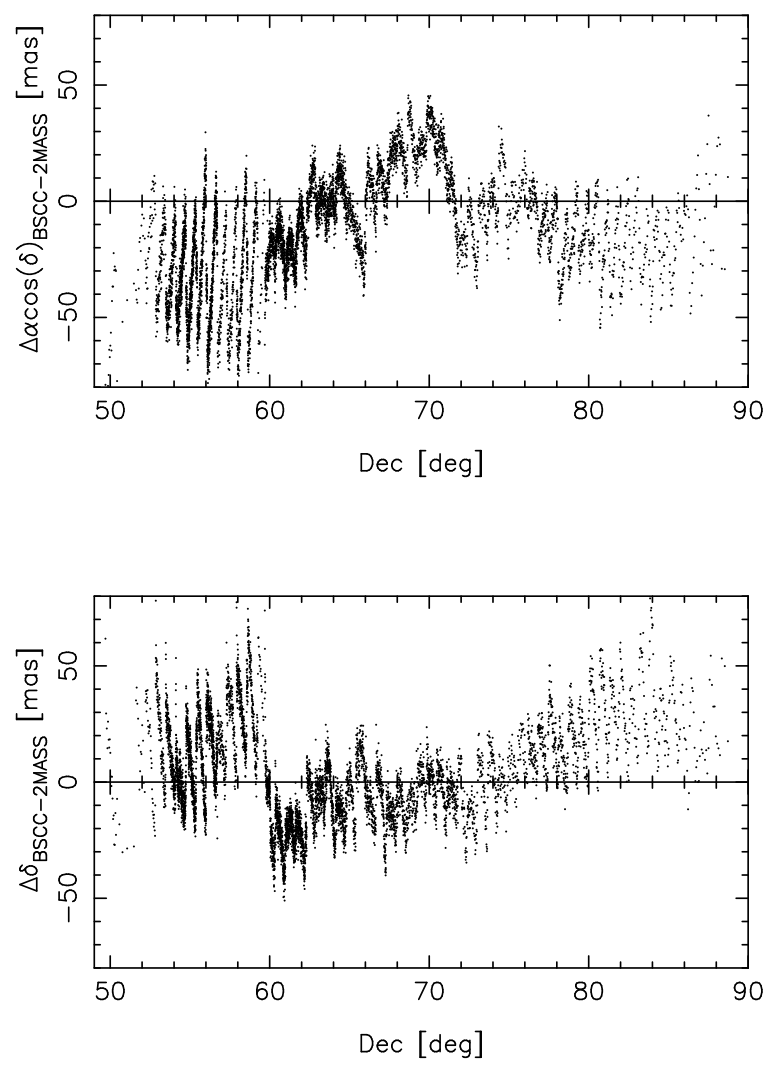

Fig. 14.- Position differences BSCC-2MASS in right ascension (top) and declination (bottom) as a function of declination. One dot represents the mean over 1500 stars.

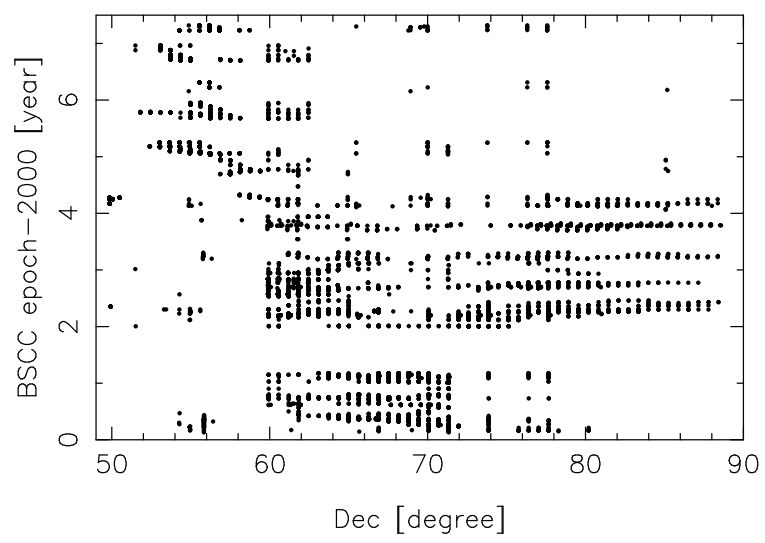

Fig. 15.- Distribution of BSCC observation epochs as a function of declination. 

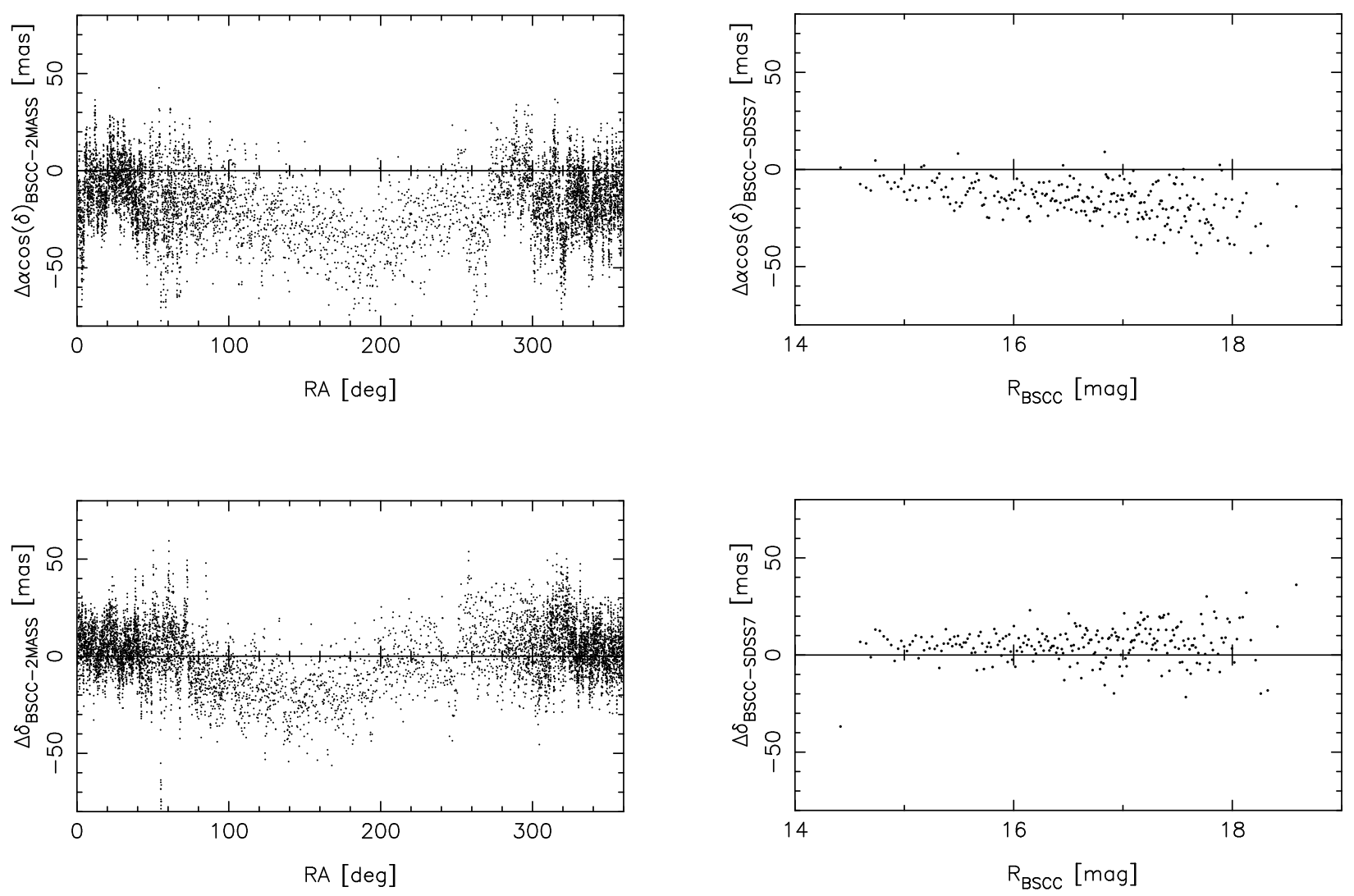

Fig. 16.- Position differences BSCC-2MASS in right ascension (top) and declination (bottom) as a function of right ascension. One dot represents the mean over 1500 stars.

Fig. 17.- Position differences BSCC-SDSS in right ascension (top) and declination (bottom) as a function of BSCC R magnitude. One dot represents the mean over 200 stars. 

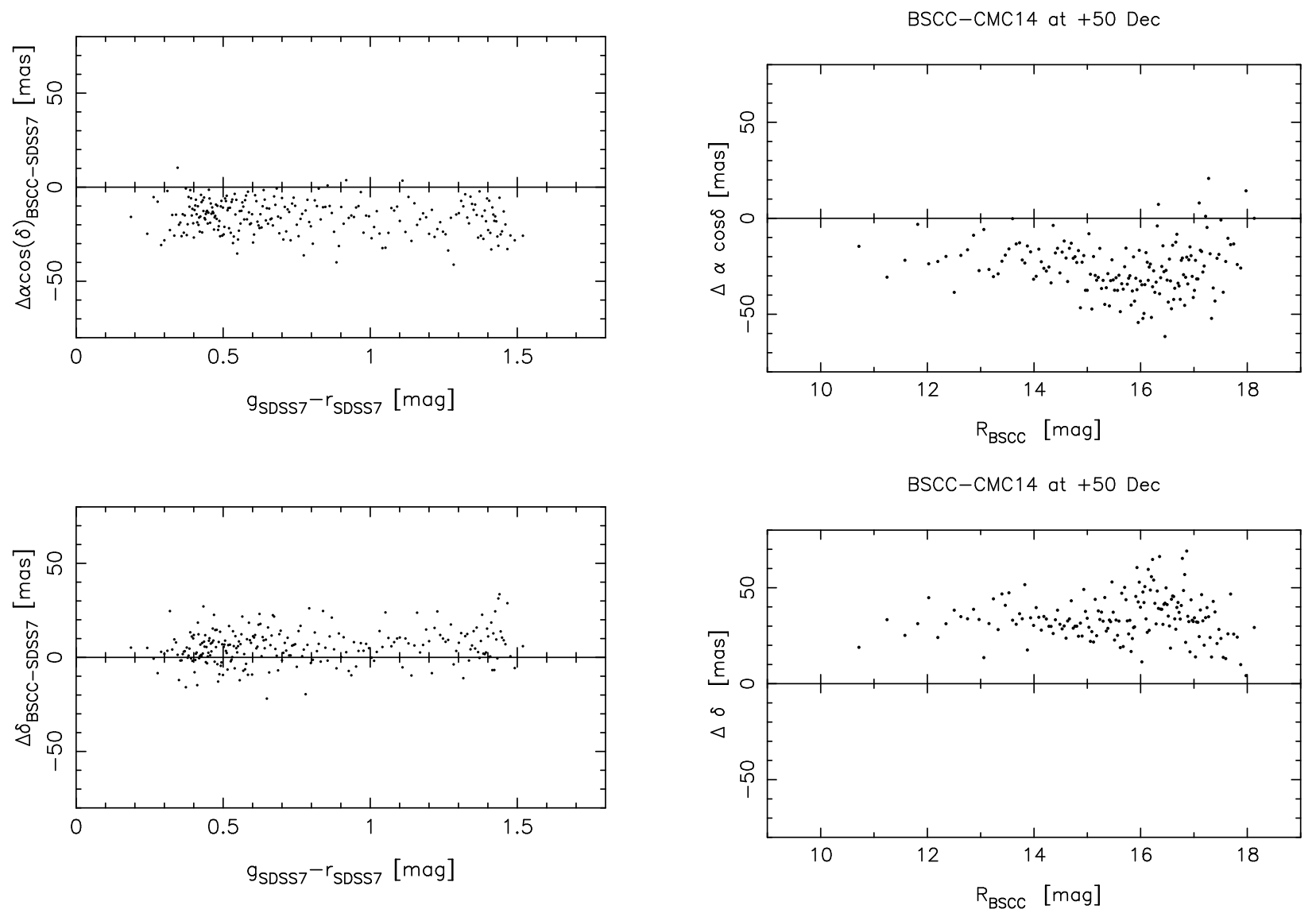

Fig. 18.- Position differences BSCC-SDSS in right ascension (top) and declination (bottom) as a function of color. One dot represents the mean over 200 stars.

Fig. 19.- Position differences BSCC-CMC14 for RA (top) and Dec (bottom) coordinate as a function of BSCC R-magnitude. One dot represents the mean over 100 stars. 

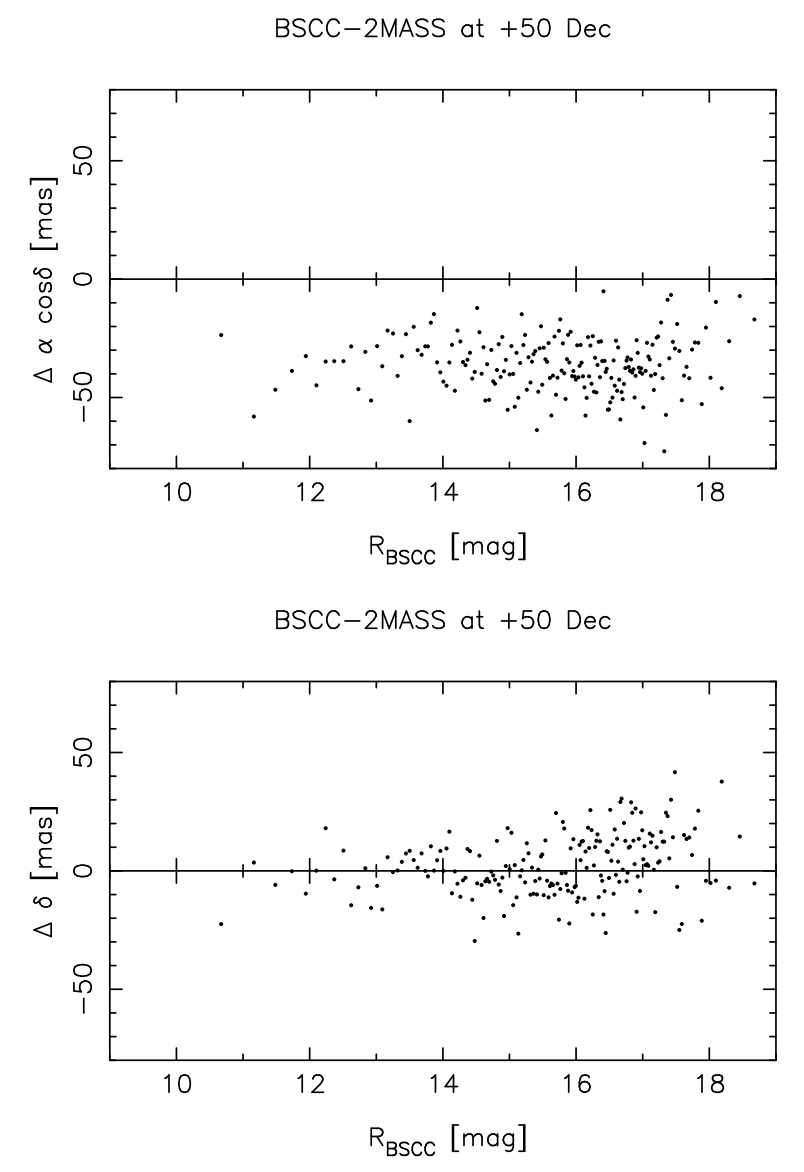

Fig. 20.- Position differences BSCC-2MASS for RA (top) and Dec (bottom) coordinate as a function of BSCC R-magnitude, for the BSCC vs. CMC14 overlap area only. One dot represents the mean over 100 stars.

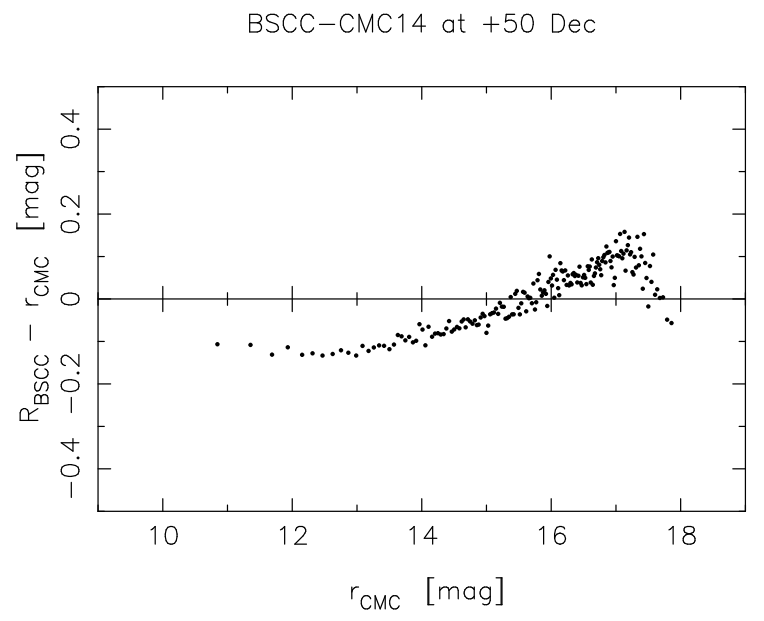

Fig. 21. - Magnitude differences BSCC R CMC14 $\mathrm{r}$ as a function of CMC14 $\mathrm{r}$ magnitude. One dot represents the mean over 100 stars after excluding outliers.

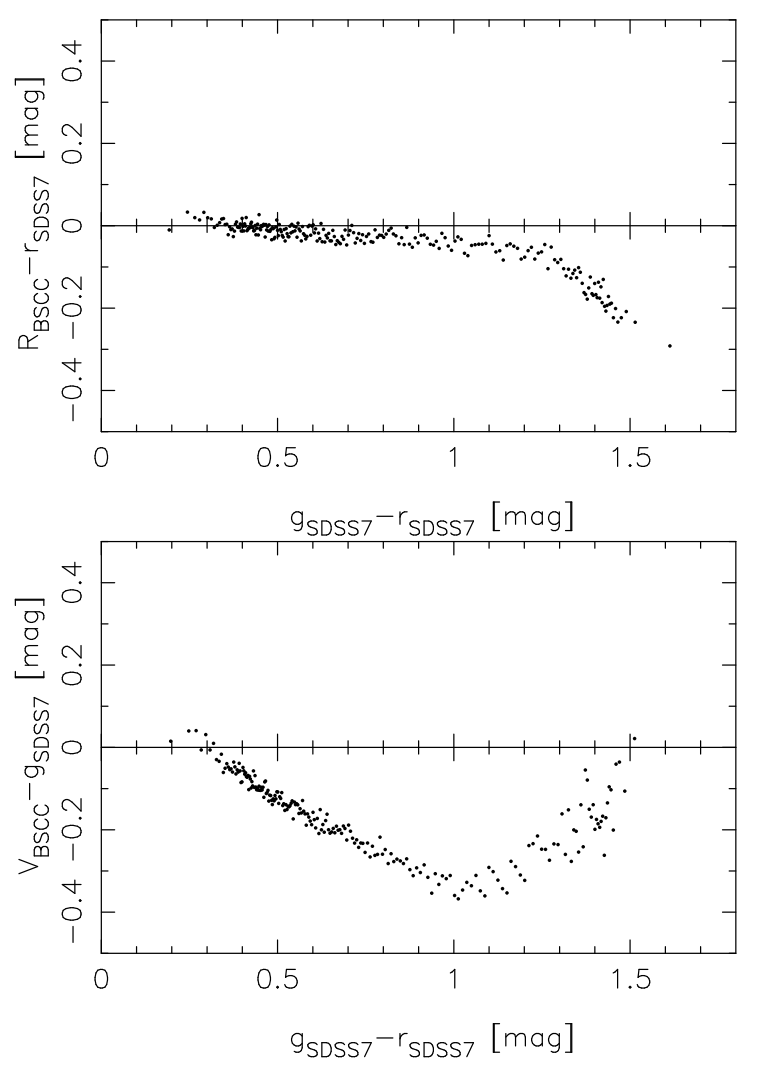

Fig. 22.- Color-Color diagrams for BSCC R magnitude (top) and $\mathrm{V}$ magnitude (bottom) as compared to SDSS $r$ and g magnitudes. One dot represents the mean over 200 stars after excluding outliers. 
Table 1: The first 10 lines of the BSCC data file.

\begin{tabular}{|c|c|c|c|c|c|c|c|c|c|c|c|c|c|c|c|c|c|c|c|c|c|c|}
\hline lumn & 2 & 3 & 4 & 5 & 6 & 789 & 10 & 11 & 12 & 13 & 14 & 15 & 16 & 17 & 18 & 19 & 20 & 21 & 22 & 23 & 24 & \\
\hline 688580578 & 8689890 & 05 & 81 & 05 & 81 & 10 & & 0 & 0 & & & 1 & 000 & -1 & 0 & 86589348 & 1367 & 25 & & 23 & & \\
\hline & & 215 & 235 & 79 & 81 & 220 & & 0 & 0 & & & 1 & & & & & & & & 82 & & \\
\hline & & 80 & 280 & 63 & 62 & 220 & & 0 & 0 & & & 1 & & & & & & & & & & \\
\hline 546 & & 313 & 59 & 122 & 121 & 220 & 4172 & 0 & 0 & & & 1 & & & & & & & & & 357 & \\
\hline & & 12 & 107 & 56 & 56 & 220 & 4251 & 0 & 0 & & - & 1 & & & & & & & & 9 & 719 & \\
\hline & & 35 & 31 & 79 & 81 & 220 & 4172 & 0 & 0 & 981 & & 1 & & - & & 850 & 029 & & & 4 & 470 & \\
\hline 129 & 017 & 229 & 229 & 229 & 229 & 110 & 4172 & 0 & 0 & 8172 & - & 1 & 000 & -1 & 0 & 395 & 15287 & & 4778 & 2 & 4460 & \\
\hline 2337 & & 30 & 88 & 61 & 61 & & 4251 & 0 & 0 & 496 & & 1 & 5866 & -1 & & & 15330 & & 788 & 2 & 8007 & \\
\hline 2559 & 5609 & 61 & 66 & 51 & & & 4251 & 0 & 0 & 335 & & 1 & 6029 & -1 & & 95487090 & 14271 & 30 & 3871 & 6 & & \\
\hline & & 14 & 24 & 53 & 52 & & & & 0 & & & & & & & & & & & & & \\
\hline
\end{tabular}


TABLE 2

Description of BSCC DATA COLUMNS AND RANGe of DATA VAlues.

\begin{tabular}{|c|c|c|c|c|c|}
\hline $\begin{array}{l}\text { BSCC } \\
\text { column }\end{array}$ & $\begin{array}{l}\text { min } \\
\text { data }\end{array}$ & $\begin{array}{l}\max \\
\text { data }\end{array}$ & $\begin{array}{c}\text { number } \\
\text { zeros }\end{array}$ & unit & $\begin{array}{l}\text { description } \\
\text { of data item }\end{array}$ \\
\hline 1 & 1491551 & 1294725510 & 0 & mas & RA (ICRS at mean \\
\hline 2 & 178689890 & 319898157 & 0 & mas & DEC epoch of obs.) \\
\hline 3 & 0 & 999 & 54401 & mas & scatter sigma $\mathrm{RA}^{*} \cos \mathrm{Dec}$ \\
\hline 4 & 0 & 999 & 89491 & mas & scatter sigma Dec \\
\hline 5 & 1 & 400 & 0 & mas & model sigma $\mathrm{RA} * \cos \mathrm{Dec}$ \\
\hline 6 & 2 & 400 & 0 & mas & model sigma Dec \\
\hline 7 & 1 & 288 & 0 & & total numb. images this star \\
\hline 8 & 1 & 283 & 0 & & numb. images used for mean pos \\
\hline 9 & 0 & 10 & 13711030 & & numb. images rejected outliers \\
\hline 10 & 132 & 7321 & 0 & $1 / 1000 \mathrm{yr}$ & mean epoch [year-2000] \\
\hline 11 & 0 & 4 & 12828714 & & largest double star flag \\
\hline 12 & 0 & 1 & 13023146 & & bad pixel flag $(1=$ bad, else 0$)$ \\
\hline 13 & 7470 & 20000 & 0 & mmag & mean $\mathrm{R}$ model fit magnitude \\
\hline 14 & -1 & 900 & 22693 & mmag & sigma R mag \\
\hline 15 & 0 & 145 & 583043 & & numb. images used for $\mathrm{R}$ mag \\
\hline 16 & 6846 & 20000 & 0 & mmag & mean V model fit magnitude \\
\hline 17 & -1 & 900 & 17299 & mmag & sigma V mag \\
\hline 18 & 0 & 144 & 4311081 & & numb. images used for $\mathrm{V}$ mag \\
\hline 19 & 0 & 1321535437 & 614483 & & 2MASS ID number \\
\hline 20 & 2069 & 30000 & 0 & mmag & 2MASS J magnitude \\
\hline 21 & -1 & 999 & 0 & mmag & 2MASS sigma $J$ magnitude \\
\hline 22 & 818 & 30000 & 0 & mmag & 2MASS H magnitude \\
\hline 23 & -1 & 999 & 0 & mmag & 2MASS sigma H magnitude \\
\hline 24 & -13 & 30000 & 0 & mmag & 2MASS K magnitude \\
\hline 25 & -1 & 999 & 0 & mmag & 2MASS sigma K magnitude \\
\hline
\end{tabular}

Note.- Undefined photometric errors are set to -1 , and a value of 30000 for a magnitude indicates "no data". 